\title{
Two-dimensional Bose gas at low density
}

\author{
A.A. Ovchinnikov \\ Institute for Nuclear Research of the Russian Academy of Sciences, \\ Moscow 117312 Russia
}

\begin{abstract}
We propose a new method to describe the interacting bose gas at zero temperature. For three-dimensional system the correction to the ground- state energy in density is reproduced. For two-dimensional dilute bose gas the ground- state energy in the leading order in the parameter $\left|\ln \alpha^{2} \rho\right|^{-1}$ where $\alpha$ is a scattering length is obtained.
\end{abstract}


At present time two-dimensional models attract much attention in connection with the problems of high- $T_{c}$ superconductivity and fractional quantum Hall effect. In this context the description of the two-dimensional system of bosons may be important. For instance the system of planar fermions in the magnetic field is equivalent to the system of the interacting bosons with the additional long-range Chern-Simons interaction. The problem of the hard core bosons on a lattice at high density is closely related to the description of different strongly correlated electronic systems. The conventional methods of the description of the interacting bose gas are inapplicable in both of the above mentioned problems. In this context the new ways to treat the system in the two different physical limits where the perturbation theory is possible are of interest.

Various methods to describe the system of bosons with the pairwise interaction were introduced which in one way or another consisted in summing up an infinite subset of the terms in the perturbation series. The example of this procedure is the Bogoliubov's method [1] which is correct in the high density limit which means that the range of the potential is much larger than the average particle spacing. In the opposite limit of the dilute bose gas the perturbation theory [1] does not work which manifests in the divergence in the expression for the energy for the $\delta$-function type potential. Various modifications of this procedure using the pseudopotential or the diagrammatic expansion methods, for example, were proposed [2]. However the reason why the procedure of ref. [1] can be applied when the two-body potential is not small and the wave function is not close to the unperturbed one is obscured. The generalization of this methods to the 2D system is not straightforward due to the behavior of the modified scattering amplitude which approaches zero in the low energy limit. In fact the methods [2] can be considered as an application of the Bogoliubov's approach to a system with the parameters chosen in such a way that both this approximation is valid and at the same time the density $\rho$ is small in a sense $\alpha \rho^{1 / d}<<1$ ( $\alpha$ is the scattering length). Although this can be a basis for the solution of 2D problem the estimate of the accuracy of the approximation [1] for a given potential is required. 
In the present paper we suggest a new method to describe the system of bosons for the two limiting cases and investigate the ground state properties of 2D bose gas at zero temperature. Our method is closely related to the approach proposed by Lieb [3] which makes use of the distribution functions related to the ground state wave function. We use the decomposition of the logarithm of the wave function into a series over the $n$ particle functions. We argue that at small density the proposed expansion corresponds to the expansion of energy in the small parameter. Although in practice it is difficult to solve the corresponding system of equations beyond the leading order approximation which in the framework of our approach corresponds to the wave function of the Jastrow form, the convincing arguments in favor of the validity of this procedure in the leading order can be found. Of course the distribution functions can be calculated using the wave function of the Jastrow form. In this way the connection with the approach of ref.[3] can be made.

The equation for the energy $E_{0}$ and the wave function $\Phi\left(x_{1}, \ldots x_{N}\right)$ of the ground state for a system of $N$ particles in the volume $V$ interacting with the two-body potential $U(x)$ has the form

$$
\left(-\sum_{i} \partial_{i}^{2}+2 \sum_{i<j} U\left(x_{i j}\right)\right) \Phi=E_{0} \Phi, \quad i, j=1, \ldots N
$$

where $\partial_{i}=\partial / \partial x_{i}, x_{i j}=x_{i}-x_{j}$ and we denote by $x_{i}$ the $d$-dimensional space vector of $i$-th particle throughout the paper. The ground state wave function $\Phi\left(x_{1}, \ldots x_{N}\right)$ is a symmetric and positive function of its arguments. According to [4] (see also [5]) one can seek for the function $\Phi$ in the form

$$
\Phi\left(x_{1}, \ldots x_{N}\right)=\exp S\left(x_{1}, \ldots x_{N}\right)
$$

We observe that the function $S\left(x_{1}, \ldots x_{N}\right)$ can be expanded as follows

$$
S\left(x_{1}, \ldots x_{N}\right)=\sum_{i<j} S_{2}\left(x_{i j}\right)+\sum_{i<k<l} S_{3}\left(x_{i}, x_{k}, x_{l}\right)+\ldots
$$


where $S_{n}\left(x_{1}, \ldots x_{n}\right)$ are the symmetric functions depending on the relative interparticle distances and subjected to the constraints

$$
\int d x_{n} S_{n}\left(x_{1}, \ldots x_{n}\right)=0, \quad n>2 .
$$

Integrating Eq.(2) over the coordinates of $N-n$ particles subsequently for $n=2,3 \ldots$ and using the condition (3) one can show that the expansion (2) is an irreducible one which means that for a given function $S\left(x_{1}, \ldots x_{N}\right)$ the set of the functions $S_{n}$ is unique. Substituting Eq.(2) into the equation (11) we obtain the equation

$$
\begin{gathered}
E_{0}=\sum_{i \neq j} F\left(x_{i j}\right)-\sum_{i \neq k \neq l} \partial S\left(x_{i k}\right) \partial S\left(x_{i l}\right)+\ldots, \\
F(x)=-\partial^{2} S(x)-\partial S(x) \partial S(x)+U(x),
\end{gathered}
$$

where the terms depending on the pair function $S_{2}(x)=S(x)$ only are indicated explicitly. Integrating Eq.(44) over the coordinates $n+1, \ldots N$ and using Eq.(3) we obtain the $n$-particle equation for the functions $S_{n}$. In general the system of the equations cannot be solved and the assumptions on the higher order $S_{n}$ - functions are required. For the dilute bose gas the perturbation theory in the small parameter can be used. Although the calculations in the high orders are too complicated one can argue that the expansion of the energy in the small parameter corresponds just to the expansion (22). Here we will demonstrate it in the lowest order which corresponds to the pairwise wave function. It is plausible that the same is valid for the higher order terms. Suppose for a while that only the function $S(x)$ is not equal to zero. Integrating the equation (4) over the coordinates $1, \ldots N$ we obtain for the parameter $a$ defined as

$$
E_{0}=N \rho a
$$

$\left(\rho=N / V, E_{0} / V=a \rho^{2}\right)$ the equation

$$
a=\frac{N-1}{N} \int d x F(x)
$$


which is equivalent to $a=\int d x\left(U-(\partial S)^{2}\right)$ in the infinite volume limit. Integrating Eq.(田) over the coordinates $3, \ldots N$ and using Eq.(河) we obtain the equation

$$
\partial^{2} S(x)+\partial S \partial S(x)-U(x)-\rho \partial_{1}^{2} \int d x_{3} S\left(x_{13}\right) S\left(x_{23}\right)=-a / V .
$$

where the notation $x=x_{12}$ is used and the terms of order $\sim 1 / V$ are retained. The right- hand side is equal to zero in the limit $V \rightarrow \infty$. We assume the periodic boundary conditions so that the integral over the total derivative is zero. Due to the condition (33) the contribution of $S_{3}$ to Eq.(河) is of the form $\rho \int d x_{1} d x_{2}\left(\partial_{1} S(123)\right)^{2}$ and the example of its contribution to Eq.(6) is $\rho \int d x_{3} \partial_{3} S(123) \partial_{3} S(13)$. One can use the solution of Eq.(66) to estimate the function $S_{3}$ with the help of the three-particle equation and show that these integrals are suppressed. We will show below that it is the smallness of $S(x)$ at the distances $\sim \rho^{-1 / d}(d=2,3)$ that allow one to neglect the $S_{3}$ function in the lowest order approximation. The equation (6) reproduce the correction in density [2] to the ground- state energy for 3D system and predicts the leading order term for $2 \mathrm{D}$ system.

Note also that Eq.(6) can be regarded as an equation for the trial variational wave function of the Jastrow form. In this case it follows from the form of the solution of Eq.(66) that the variational energy is given by Eq.(5) with the accuracy up to the higher order terms in the expansion parameter.

If the second term in the left-hand side of the equation (6) can be neglected then the equation can be easily solved using the Fourier transformation:

$$
\rho S_{k}^{2}-k^{2} S_{k}-U_{k}=0, \quad S_{k}=\int d x e^{i k x} S(x) .
$$

Substituting the solution of this equation $S_{k}$ to the expression for the energy

$$
a=U_{0}+\int d x(\partial S(x))^{2}=U_{0}+\int_{k} k^{2} S_{k}^{2},
$$

$\left(U_{0}=\int d x U(x)\right)$ we get

$$
a=U_{0}+\frac{1}{2 \rho^{2}} \int_{k}\left(\left(k^{4}+4 U_{k} \rho k^{2}\right)^{1 / 2}-k^{2}-2 U_{k} \rho\right),
$$


where the notation $\int_{k}=\int d^{d} k /(2 \pi)^{d}$ is used. Eq.(7) is the Bogoliubov's expression for the energy. One can estimate the accuracy of Eq.(77) for a given two-body potential $U(x)$. The corrections are determined by the function $S(x)$ which should be small in order the approximation (7) to be valid. For instance in 2D for the potential which is a repulsive square well in momentum space, $U_{k}=u, k<b^{-1}, U_{k}=0, k>b^{-1}$, the energy is: $(i)$ at $u \rho b^{2}>>1, a=u\left(1-\frac{1}{4 \pi} \frac{1}{\rho b^{2}}\right), S(0)=-\left(u / \rho b^{2}\right)^{1 / 2} / 2 \pi$; (ii) at $u \rho b^{2}<<1, a=u\left(1-\frac{u}{4 \pi} \ln \left(u \rho b^{2} e^{1 / 2}\right)\right), S(0)=-1 / 8 \pi \rho b^{2}$. Briefly, the potential should be relatively shallow compared to its width and the spatial range should be much larger than the average particle spacing.

Let us proceed with the solution of the equation (6) in the low density limit. The particle density is assumed to be small so that the dimensionless parameter $\alpha \rho^{1 / d}<<$ 1. In this formula $\alpha$ is the scattering length for the potential $U$ and $\rho^{-1 / d}$ is the average interparticle distance in $d$ - dimensional space. The expansion parameters are respectively $\left|\ln \rho \alpha^{2}\right|^{-1}$ and $\alpha^{3 / 2} \rho^{1 / 2}$ in two and three dimensions.

\section{Three-dimensional system.}

Let us begin with the case of three spatial dimensions $d=3$. Define $S_{0}(x)$ as the solution of the equation (6) at $\rho=0$ :

$$
\partial^{2} S_{0}+\partial S_{0} \partial S_{0}-U=-\alpha / V
$$

Here $\alpha$ is the unknown parameter and the periodic boundary conditions are implied. In terms of the function $\phi_{0}(x)=\exp \left(S_{0}(x)\right)$ keeping the volume finite and taking into account the periodic boundary conditions we find that

$$
\alpha=\int d x\left(U(x)+S_{0} \partial^{2} S_{0}(x)\right)=\int d x U(x) \phi_{0}(x)
$$

is proportional to the scattering length for the potential $U(x)$. For the hard-sphere potential $\alpha$ is the radius of the potential. In the infinite volume limit the equation has the form $\left(-\partial^{2}+U\right) \phi_{0}(x)=0$ which is to be supplemented by the boundary 
condition $\phi_{0}(r) \rightarrow 1$ at $r \rightarrow \infty(r=|x|)$. At the distances much larger than the range of the potential the solution is $\phi_{0}(r)=1-\alpha / r$. For the energy we have $a=$ $\alpha+\int d x\left(S \partial^{2} S-S_{0} \partial^{2} S_{0}\right)$ or, equivalently,

$$
a=\alpha-\int_{k}\left(k^{2} S_{k}^{2}-k^{2} S_{0 k}^{2}\right)
$$

Make use of the Fourier transformation in the equations (6) and (8). In the region

$r>>\alpha$ where the condition $S_{0}(x)<<1$ is satisfied we have $S_{0}(r)=-\alpha / r$ and the main contribution to the integral $\int d x\left(\partial S_{0}\right)^{2}$ comes from the integration over the region of the small $r \sim \alpha$. Up to the corrections in $\rho$ the same is true for the analogous integral for $S(x)$.

Therefore the Fourier transform of the function $U-(\partial S)^{2}$, and the same function for $S_{0}$, are independent of the momentum $k$ at $k<<1 / \alpha$. At these values of $k, S_{0 k}=$ $-\alpha / k^{2}$ and $S_{k}$ is given by the solution of the equation $\rho k^{2} S_{k}^{2}-k^{2} S_{k}-\alpha=0$. Substituting these functions into Eq.(9) we obtain

$$
a=\alpha+\frac{1}{2 \rho^{2}} \int_{k}\left(\sqrt{k^{4}+4 \alpha \rho k^{2}}-k^{2}-2 \alpha \rho+\frac{2 \alpha^{2} \rho^{2}}{k^{2}}\right)
$$

The integral in this expression converges at large $k$ and is saturated at the values $k \sim(a \rho)^{1 / 2}<<1 / \alpha$ which justifies our assumption that $\int d x e^{i k x}\left(U-(\partial S)^{2}\right)=\alpha$. The corrections to this formula does not change the result in the approximation considered. Evaluating the integral in Eq.(10) we obtain the well known result [2] for the expansion of the ground state energy in powers of density in $3 \mathrm{D}$ :

$$
a=\alpha\left(1+\frac{16}{15 \pi^{2}} \alpha^{3 / 2} \rho^{1 / 2}\right) .
$$

\section{Two-dimensional system.}

Let us proceed with the investigation of two-dimensional system. In 2D it is easy to find the solution of Eq.(6) and determine the energy $a$ as the function of the density $\rho$ with the accuracy up to the terms of the higher order in the small parameter $a \sim$ $\left|\ln \rho \alpha^{2}\right|^{-1}$. 
As in three-dimensional case the solution of Eq.(可) can be represented in the form

$$
S_{k}=-\frac{1}{2 \rho}\left(\sqrt{1+4 \sigma_{k} \rho / k^{2}}-1\right)
$$

where $S_{k}$ is the Fourier transform of the function $S(x)$ and the function $\sigma_{k}$ is defined by

$$
\sigma_{k}=\int d x e^{i k x}(U(x)-\partial S \partial S(x))
$$

At the sufficiently small $k$ we have $\sigma_{k}=a$ while at the larger $k, \sigma_{k}$ could depend on the momentum $k$. However it can be shown that in fact with the accuracy of order $O\left(a^{2}\right)$ one can take $\sigma_{k} \simeq a$ for all $k<<\alpha^{-1}$. We are interested in the behavior of the function $S(x)$ in the region $\alpha<<r<<(a \rho)^{-1 / 2}$. As in 3D $(a \rho)^{-1 / 2}$ is the characteristic length, the kind of correlation length in the problem. In this region $S(x)$ can be calculated as

$$
S(x)=-\frac{1}{2 \rho} \int_{k} e^{i k x}\left(\left(1+\frac{4 a \rho}{k^{2}}\right)^{1 / 2}-1\right)
$$

Evaluating this expression we find that

$$
S(x)=-a / 4 \pi+(a / 2 \pi) \ln \left((a \rho)^{1 / 2} r\right)
$$

which is valid in the indicated region with the accuracy up to the terms of order of $a^{2}$. One can see from Eq.(12) that the function $S(x)$ decreases quickly at the distances much larger than the correlation length $(a \rho)^{-1 / 2}$ :

$$
S(x) \simeq-\frac{1}{2 \pi}\left(\frac{a}{\rho}\right)^{1 / 2} \frac{1}{r}, \quad r>>(a \rho)^{-1 / 2} .
$$

Now using the equation (13) one can estimate the function $\sigma_{k}$ :

$$
\left|\sigma_{k}-a\right|<\frac{a^{2}}{2 \pi} \ln \frac{k}{\sqrt{a \rho}}+O\left(a^{2}\right) .
$$

This relation justifies the initial assumption about the behavior of $\sigma_{k}$. The other way to obtain the solution (13) is to represent the equation (6) in the form

$$
\left(\partial^{2}-U(x)\right) \phi(x)=\rho \phi(x) \partial_{1}^{2} \int d x_{3} S\left(x_{13}\right) S\left(x_{23}\right)-(a / V) \phi(x)
$$


where $\phi(x)=\exp S(x)$. The Fourier transform of the function $U(x) \phi(x)$ does not depend on the momentum at $k<<1 / \alpha$. In the large distance region where $S(x)<<1$ we can expand the function $\phi(x) \simeq 1+S(x)$ and neglect the terms of order $S^{2}(x)$. Then the equation (14) has the solution of the same form as Eq.(13). The only difference is that the parameter $a$ is replaced by the value of the integral $\int d x U(x) \phi(x)$. To calculate it one has to integrate both sides of the equation (14) over the space vector in the finite volume $V$ :

$$
\begin{gathered}
\int d x U(x) \phi(x)=a-\rho \int d x(1-\phi(x)) \chi(x) \\
\chi\left(x_{12}\right)=\partial_{1}^{2} \int d x_{3} S\left(x_{13}\right) S\left(x_{23}\right)
\end{gathered}
$$

In general the limit $V \rightarrow \infty$ of integral of some function over the volume does not coincide with the integral of the limiting function, because in the finite volume the function may have the asymptotic const./V. This is indeed the case for $\int d x \chi(x)$ which is zero in the finite volume. However one can substitute the product of the limiting functions $(1-\phi(x)) \chi(x)$ into Eq.(15) since both functions approaches zero at $r \rightarrow \infty$. Alternatively, one can use the equation (6) directly in the infinite volume limit. The function $\chi(r)$ can be evaluated using Eq.(12):

$$
\chi(r) \simeq a S(r),\left(\alpha<<r<<(a \rho)^{-1 / 2}\right) ; \quad \chi(r) \simeq \frac{1}{2 \pi}\left(\frac{a^{1 / 2}}{\rho^{3 / 2}}\right) \frac{1}{r^{3}},\left(r>>(a \rho)^{-1 / 2}\right) .
$$

The integral in the right hand side of the equation (15) is determined by the longdistance region $(S(x)<<1)$ and can be estimated as $O\left(a^{2}\right)$. Thus the solution of Eq.(6) as a function of the parameter $a$ is found.

On the other hand at the sufficiently small $r$ the function $\phi(x)$ can be found approximately as the solution of the equation $\left(-\partial^{2}+U(x)\right) \phi(x)=0$ due to the fact that the right hand side of Eq.(14) is $\sim \rho$. The solution is $\phi_{0}(r)=C \ln (r / \alpha)$ where $C$ is an arbitrary constant and $\alpha$ is the scattering length for the potential $U(r)$ (the region $r>>\alpha$ is implied). In two dimensions the scattering length is defined by the behavior 
of the scattering amplitude at low energy which (for our equation) is given by

$$
\bar{f}(k)=\frac{\pi}{\ln k \alpha / 2+\gamma-i \pi / 2}+O(k \alpha),
$$

where $|\bar{f}(k)|=(2 \pi k)^{1 / 2}|f(k)|$ is the modified scattering amplitude, $k$ is the momentum and $\gamma=0.1159$ is the Euler constant. For the 2D hard-sphere potential the scattering length $\alpha$ is equal to the radius of the potential. The correction $\delta \phi(r) \sim a \rho r^{2}$ to the solution of the homogeneous equation can be easily estimated using Eq.(13). The correction is $\sim 1$ at the distances of order of the correlation length $(a \rho)^{-1 / 2}$ however it is small $(\delta \phi(r) \sim a)$ at the distances $r \sim \rho^{-1 / 2}$. At $r \sim \rho^{-1 / 2}$ the equation (13) is still valid. Comparing the solution given by Eq.(13) with the function $\phi_{0}(r)$ we get the relation

$$
C \ln (r / \alpha)=1-a / 4 \pi+(a / 2 \pi) \ln \left((a \rho)^{-1 / 2} r\right)
$$

which should be valid in the region $\alpha<<r<<(a \rho)^{-1 / 2}$ with the logarithmic accuracy. In particular at $r \sim \rho^{-1 / 2}$ it is valid with the accuracy up to the terms of order $\sim a$. Thus in the leading order in the small parameter $\left|\ln \rho \alpha^{2}\right|^{-1}, C=a / 2 \pi$ and the energy is

$$
a=\frac{4 \pi}{\left|\ln \rho \alpha^{2}\right|}+O\left(\frac{1}{\left|\ln \rho \alpha^{2}\right|^{2}}\right) .
$$

Eq.(17) is our final result for the two- dimensional system.

Naively, from the three-particle equation the function $S_{3}$ can be estimated by an order of magnitude as $S(1,2,3) \sim S(13) S(23)+$ permutations. Then the contribution of $S_{3}$ to Eq.(可) at the distances $\sim \rho^{-1 / 2}$ which is determined by the integral $\rho \int d x_{3} S(13)\left(\partial_{1} \partial_{3}-\partial_{3}^{2}\right) S(123)$ (see Eq.(3)) is suppressed due to the smallness of the function $S(x)$ at these distances. Actually the three-particle equation can be solved in the leading order in the expansion parameter (that means that the terms $\sim S_{2} S_{3}$ in this equation should be neglected) in the momentum representation and the corresponding integral in the momentum space can be estimated (see Appendix).

At the same time at short distances where the two particles interact strongly the pair function is not expected to be very different from the solution of the two-body problem 
whether or not the function $S_{3}$ is taken into account. In the other words although the function $S_{3}$ is not necessary small at short distances our estimate of the right hand side of Eq.(14) as well as the equation (16) are valid by an order of magnitude. Note that the "momentum" corresponding to the two-particle equation is of order of the correlation length which characterizes the screening of the pair wave function due to the other particles. Hence the result (17) has a simple physical interpretation. Namely the energy of the two particles interacting with the potential $U$ located in the twodimensional volume $V$ is $\sim \bar{f} / V$ where the modified scattering amplitude $\bar{f}$ should be normalized at $k \sim \rho^{1 / 2}$. Multiplying the result by the number of pairs we obtain the result (17). In three dimensions that corresponds to the first term in Eq.(11).

The other approach to the description of the dilute bose gas based on the equations for the distribution functions related to the function $\Phi$ was proposed in ref. [3]. We will show below that the assumptions made in ref. [3] can be justified in the framework of our approach. Let us describe briefly this method. The $n$-particle distribution function is defined by

$$
\begin{gathered}
g_{n}\left(x_{1}, \ldots x_{n}\right)=Z^{-1} V^{n} \int d x_{n+1} \ldots d x_{N} \Phi\left(x_{1}, \ldots x_{N}\right) \\
Z=\int d x_{1} \ldots d x_{N} \Phi\left(x_{1}, \ldots x_{N}\right) .
\end{gathered}
$$

The energy is related to the pair distribution function $g_{2}\left(x_{12}\right)=g(x)$ by means of

$$
a=\frac{N-1}{N} \int d x U(x) g(x) .
$$

Using Eq.(1) we obtain the equation for $g(x)$ :

$$
\begin{gathered}
\left(-\partial^{2}+U(x)\right) g(x)=\frac{1}{2} E_{0} g(x)-\rho \int d x_{3} g_{3}(1,2,3) U\left(x_{23}\right) \\
-\frac{1}{2} \frac{(N-2)(N-3)}{V^{2}} \int d x_{3} d x_{4} g_{4}(1,2,3,4) U\left(x_{34}\right) .
\end{gathered}
$$

The first and the third terms in the right- hand side are of order $\sim N$ while the lefthand side is of order $\sim 1$. In the leading order the cancellation of these terms take place and the corrections $\sim 1 / N$ are important. The approximation for the distribution 
functions $g_{3}$ and $g_{4}$ used in ref.[3] to evaluate the right hand side of Eq.(20) is the superposition approximation:

$$
g_{n}\left(x_{1}, \ldots x_{n}\right)=\prod_{i<j}^{n} \tilde{g}\left(x_{i j}\right)
$$

From physical considerations it is clear that in the limit $V \rightarrow \infty$ the function $\tilde{g}(x) \rightarrow$ $g(x)$ while for the finite volume it can be found using the set of the equations

$$
\int d x_{n} g_{n}\left(x_{1}, \ldots x_{n}\right)=V g_{n-1}\left(x_{1}, \ldots x_{n-1}\right)
$$

For instance, for the four- particle function $g_{4}$, substituting the ansatz (21) into the formula $\int d x_{3} d x_{4} g_{4}(1,2,3,4)=V^{2} g\left(x_{12}\right)$ we find that the corresponding pair function is

$$
\tilde{g}^{(4)}(x)=g(x)\left(1-\frac{1}{V} \int d x_{3} f\left(x_{13}\right) f\left(x_{23}\right)\right),
$$

where the function $f(x)=1-g(x)$ can be taken in the infinite volume limit. These corrections are not important in the second term of the right- hand side of Eq.(20). Substituting the expressions for the functions $g_{3}$ and $g_{4}$ into the equation (20) and taking the limit $V \rightarrow \infty$ we get an equation for the pair distribution function $g(x)$. Assuming that the function $f(x)$ is small and retaining the terms of the leading order in $f(x)$ we get

$$
\left(-\partial^{2}+U(x)\right) g(x)=2 a \rho f(x)-a \rho^{2} \int d x_{3} f\left(x_{13}\right) f\left(x_{23}\right)
$$

This equation is valid for those $r$ where the function $f(x)$ is small $(f(x)<<1)$.

The equation (22) can be solved in the same way as Eq.(6). For instance the solution of Eq.(22) for 2D problem in the region where $f(x)<<1$ is

$$
g(x)=1+a / 4 \pi+(a / 2 \pi) \ln \left((a \rho)^{1 / 2} r\right)
$$

for $r<<(a \rho)^{-1 / 2}$. Repeating the arguments leading to Eq.(16) we obtain the result (17). 
In 3D the solution of the equation (22) at the distances $r<<(a \rho)^{-1 / 2}$ is

$$
g(x)=\left(1+\frac{16}{15 \pi^{2}} \alpha^{3 / 2} \rho^{1 / 2}\right)-\frac{a}{r} .
$$

Comparing this function with the solution of the homogeneous equation $\phi_{0}(r)=C(1-$ $\alpha / r)$ which is valid with the accuracy $\sim \alpha^{3 / 2} \rho^{1 / 2}$ at $r<\rho^{-1 / 3}$ we obtain the result (11).

Let us comment on the relation of our approach to that of ref. [3]. The function $g(x)$ can be calculated using the obtained wave function of the Jastrow form. Formally the problem is similar to the calculation of the distribution functions of the classical liquid. Although the expansion in density for the corresponding partition function is not valid (integral $\int d x S(x)$ diverges at large distances) the formulas of ref.[3] resulting in the Eq.(22) can be obtained in the lowest order in the expansion parameter. One can establish the direct correspondence between the equations (6) and (22). The function $g(x)$ at long distances can be calculated by means of the cluster expansion (for instance, see [7]). The connected diagrams with the lines corresponding to the function $\phi(x)-1$ between the points 1 and 2 are considered. First, the Fourier transform of the function $\phi(x)-1$ may be replaced by $S_{k}$. In the integrals corresponding to the diagrams of the cluster expansion the long distances where the function $S(x)$ is small are important. Therefore only the diagrams that does not contain the two different paths connecting the points 1, 2 are required. These diagrams can be refered to as a "chain" diagrams (see Fig.1). Adding once more line to each of these diagrams leads to an extra suppression. Since $S(r) \rightarrow 0$ at $r \rightarrow \infty$, it is clear that the sum of the "chain" diagrams gives the exact result for the asymptotic of the distribution function. The "chain" diagrams can be summed up in the momentum representation. We obtain the following relation:

$$
f_{k}=-\frac{S_{k}}{1-\rho S_{k}}
$$

Substituting the solutions of the equations (6) and (22) into Eq.(24) we find that this relation is valid with the accuracy required for the estimates (11) and (17).

The same approximation can be used to calculate the Feynman structure factor $F(k)$ which determines the energy of the low-lying excitations (phonons). In this case 
the distribution function related to the square of the wave function $\Phi$ (defined as in Eq.(18)) should be calculated. We find

$$
F(k)=\rho \frac{1}{1-2 \rho S_{k}} .
$$

In contrast to the other wave functions of the Jastrow form used in the variational studies of the bose liquid [6] we obtain the correct behavior $F(k) \sim k$ at $k \rightarrow 0$ due to the behavior $S(r) \sim 1 / r^{d-1}$ at the asymptotically large distances [7] (for a related discussion see ref.[8]). The phonon energy

$$
\omega(k)=\frac{\rho k^{2}}{F(k)}=\sqrt{k^{4}+4 a \rho k^{2}}
$$

is in agreement with the predictions of the other approaches [1], 2]. The momentum distribution $n_{k}$ can be readily calculated. In terms of the wave function it has the form

$$
n_{k}=N \int d x e^{i k x} \int d x_{2} \ldots d x_{N} \Phi\left(x, x_{2} \ldots x_{N}\right) \Phi\left(0, x_{2} \ldots x_{N}\right)
$$

$\left(\int_{k} n_{k}=\rho\right.$, the normalization $<\Phi \mid \Phi>=1$ is implied). The result is as follows

$$
n_{k}=\frac{\rho^{2} S_{k}^{2}}{1-2 \rho S_{k}}=\frac{1}{2}\left(\frac{k^{2}+2 a \rho}{\sqrt{k^{4}+4 a \rho k^{2}}}-1\right)
$$

which coincides with the known expression [1, 2]. In two dimensions the expression (17) should be substituted for $a$. In $2 \mathrm{D}$ the condensate is $N_{0}=N\left(1-\frac{1}{4 \pi} a\right)$.

Finally, the situation is different for one-dimensional problem. From the point of view of our method the low density limit coincide with the weak coupling limit. In this case the expression (7) is correct in the weak interaction limit regardless of the shape of the potential while for the strong coupling the perturbation theory is inapplicable which is in agreement with the exact solution of the problem for the $\delta$-function potential [9]. The same can be true for the lattice system. For instance for a system described by the Hamiltonian (for example, see ref. [10])

$$
H=-t \sum_{<i j>}\left(b_{i}^{+} b_{j}+\text { h.c. }\right)+U \sum_{i} n_{i}\left(n_{i}-1\right), \quad n_{i}=b_{i}^{+} b_{i}
$$


at $U \rightarrow 0$ and the density of order of unity Eq.(17) is asymptotically exact in any dimensions.

In conclusion, we presented the method to describe the interacting bose gas at zero temperature. The expansion in the irreducible functions for a logarithm of the ground state wave function was used. For a low density the equation for a Jastrow wave function was solved. It was argued that the contribution of the three-particle component of the wave function to the equation is suppressed. For three-dimensional system the leading order correction for the ground state energy in particle density was reproduced. For the two-dimensional dilute bose gas the ground state energy in the leading order in the parameter $\left|\ln \alpha^{2} \rho\right|^{-1}$ where $\alpha$ is two-dimensional scattering length was obtained.

\section{Aknowledments}

The author is grateful to D.T.Son for discussions. This work was supported, in part, by a Soros Foundation Grant awarded by the American Physical Society.

\section{Appendix}

Here we estimate the contribution of the three-particle function $S_{3}\left(x_{1}, x_{2}, x_{3}\right)$ to the equation (6). The three- particle equation is obtained after the integration of the basic equation (11) over the coordinates $4, \ldots N$. It is necessary to take into account the

equations (5) and (6) for the terms that depend only on one of the variables $x_{12}, x_{13}, x_{23}$. In the coordinate space the equation is

$$
\begin{gathered}
\frac{1}{2}\left(\partial_{1}^{2}+\partial_{2}^{2}+\partial_{3}^{2}\right) S_{3}(1,2,3)+\partial_{1} S(12) \partial_{1} S(13)+\partial_{2} S(21) \partial_{2} S(23) \\
+\partial_{1} S(12) \partial_{1} S(13)+\ldots=\frac{1}{V \rho}(F(12)+F(13)+F(23))
\end{gathered}
$$

where the dots stands for the terms of order $\sim S_{2} S_{3}, S_{3}^{2}$. These terms are not important for the solution of Eq.(25) in the leading order. The right-hand side is equal to zero in 
the infinite volume limit. In the momentum representation the function $S\left(k_{1}, k_{2}, k_{3}\right)$ is defined as

$$
\int d x_{1} d x_{2} d x_{3} e^{i k_{1} x_{1}+i k_{2} x_{2}+i k_{3} x_{3}} S(1,2,3)=(2 \pi)^{d} \delta^{d}\left(k_{1}+k_{2}+k_{3}\right) S\left(k_{1}, k_{2}, k_{3}\right) .
$$

The solution of the equation (25) is

$$
S\left(k_{1}, k_{2}, k_{3}\right)=-\frac{2}{k_{1}^{2}+k_{2}^{2}+k_{3}^{2}}\left(k_{1} k_{2} S_{k_{1}} S_{k_{2}}+k_{1} k_{3} S_{k_{1}} S_{k_{3}}+k_{2} k_{3} S_{k_{2}} S_{k_{3}}\right) .
$$

The contribution of the function $S_{3}$ to the left-hand side of Eq.(6) has the following form:

$$
2 \rho \int d x_{3} S(13)\left(\partial_{1} \partial_{3}-\partial_{3}^{2}\right) S_{3}(1,2,3)
$$

which is the function of the variable $x_{12}=x$. Substituting the solution (26) to the integral in the momentum space corresponding to the Fourier transform of the function (27) we obtain the following expression:

$$
2 \rho \int_{p} \frac{2 p^{2}-k p}{p^{2}+k^{2}-k p} S_{p}\left(k(k-p) S_{k} S_{k-p}+p(p-k) S_{p} S_{k-p}+k p S_{k} S_{p}\right),
$$

where $k$ is the external momentum. It is possible to estimate the integral of Eq.(28) at different values of the momentum $k$ with the help of Eq.(12). For example at $k=0$ we have

$$
4 \rho \int_{p} p^{2} S_{p}^{3}
$$

This integral is easily estimated as $O\left(a^{2}\right)$ and $O\left(a\left(a^{3 / 2} \rho^{1 / 2}\right)\right)$ respectively in two- and three- dimensional space. Analysing Eq. 28) it is easy to show that these estimates are valid at arbitrary $k$. Therefore the considered contribution does not change result in the approximation required for the derivation of the formulas (11) and (17).

\section{References}

[1] N.N.Bogoliubov, J.Phys.(Moscow) 11 (1947) 23. 
[2] T.D.Lee, C.N.Yang, Phys.Rev. 105 (1957) 1119. T.D.Lee, K.Huang, C.N.Yang, Phys.Rev. 106 (1957) 1135. S.T.Beliaev, Zh.Exp.Teor.Phys. 33 (1958) 289. K.A.Brueckner, K.Sawada, Phys.Rev. 106 (1957) 1117.

[3] E.H.Lieb, Phys.Rev. 130 (1963) 2518.

[4] A.Bijl, Physica 7 (1940) 869. R.Jastrow, Phys.Rev. 98 (1955) 1479.

[5] N.N.Bogoliubov, D.N.Zubarev, Zh.Exp.Teor.Phys. 28 (1955) 129.

[6] W.L.McMillan, Phys.Rev. 138 (1965) A442. J.B.Aviles, Ann.Phys.(NY) 5 (1958) 251. K.Hiroike, Progr.Theor.Phys. 27 (1962) 342. L.Reatto, Phys.Rev. 183 (1969) 334. W.P.Francis et.al., Phys.Rev. A1 (1970) 86.

[7] L.Reatto, G.V.Chester, Phys.Rev. 155 (1967) 88.

[8] C.L.Kane, S.Kivelson, D.H.Lee, S.C.Zhang, Phys.Rev.B 43 (1991) 3255.

[9] E.H.Lieb, W.Liniger, Phys.Rev. 130 (1963) 1605.

[10] D.S.Rokhsar, B.G.Kotliar, Phys.Rev.B 44 (1991) 10328. 